RESEARCH PAPER

\title{
Smoking behaviour and toxin exposure during six weeks use of a potential reduced exposure product: Omni
}

\author{
J R Hughes, S S Hecht, S G Carmella, S E Murphy, P Callas
}

Tobacco Control 2004;13:175-179. doi: 10.1136/tc.2003.005439

See end of article for authors' affiliations

....................

Correspondence to: John R Hughes,

$M D$, University of Vermont, Departments of Psychiatry, Psychology and Family

Practice, 38 Fletcher Place, Burlington, VT 05401-1419, USA; john.

hughes@uvm.edu

Received 24 July 2003

Accepted 3 January 2004

\begin{abstract}
Objective: To determine smoking behaviour, acceptability, and toxin exposure when smokers switch to the potential reduced exposure product-Omni cigarette.

Design: 12 week randomised, crossover study of Omni versus own cigarettes.

Participants: 19 light/ultralight and 15 regular smokers.

Outcomes: Cigarettes/day, smoking topography, craving, withdrawal symptoms, urinary cotinine plus its glucuronide (total cotinine), nicotine plus its glucuronide (total nicotine), and carcinogen metabolites (4-(methylnitrosamino)-1-(3-pyridyl)-1-butanol plus its glucuronides and 1-hydroxypyrene).

Results: When switched to Omni, smokers smoked the same number of cigarettes/day, smoked Omni cigarettes less intensely (total puff volume $=-11 \%$ ) and had slightly lower total cotinine $(-18 \%$ ) levels than their own cigarettes, but had a slightly greater carbon monoxide boost/cig (+21\%). Craving and withdrawal ratings were similar with Omni and own cigarettes. Carcinogen metabolite levels were somewhat but not significantly lower with Omni. About half of smokers rated Omni as better for their health and about two thirds stated it was weaker and worse tasting than their own cigarettes.

Conclusions: Although Omni may be an adequate behavioural and pharmacological substitute for traditional cigarettes, it may not decrease carcinogen exposure and may increase carbon monoxide. Replications with larger sample sizes and longer follow up are needed. These results indicate the need for regulation of reduced exposure and reduced risk claims.
\end{abstract}

$\mathrm{H}$ arm reduction is usually defined as non-cessation methods to reduce the harm from drug use. ${ }^{12}$ One of the several methods of harm reduction for tobacco use $^{1-5}$ is to change the tobacco product. Tobacco and nontobacco companies have marketed or plan to market "potential reduced exposure products (PREPs) ${ }^{2}$ to reduce the exposure or risk from tobacco use. Such products that are cigarettes include Advance, Next, Omni, Quest (Omni-Free), and Scor. Unfortunately, due to the absence of governmental regulation, whether these products actually reduce exposure or risk is relatively untested. ${ }^{1-4}$

The current study examined carcinogen, nicotine, and carbon monoxide exposure, smoking behaviour, and pharmacological substitutability of one of the more recent and more widely promoted PREPs-Omni (www.omnicigs.com). Omni is a traditional cigarette in which the tobacco has been treated with the catalyst palladium to improve its burning efficiency. ${ }^{67}$ Since many carcinogens are the result of incomplete combustion, ${ }^{8}$ this would be expected to reduce carcinogen exposure. Omni has recently been marketed in the USA with claims of reduced carcinogen exposure. Omni ads state: "The three groups of carcinogens that have been significantly reduced are polycyclic aromatic hydrocarbons (PAHs), tobacco specific nitrosamines (TSNAs) and catechols. PAHs, TSNAs, and catechols are among the most potent and dangerous substances in tobacco smoke in relation to lung cancer incidence." (www.omnicigs.com). Omni cigarettes differ from the recently released Quest (aka Omni-Free) cigarettes from the same company in that the Quest cigarettes are reduced nicotine cigarettes but the Omni cigarettes are not.

The Omni website reports the levels of several toxins in machine tests comparing Full-Flavor Omnis and Light Omnis versus "leading competitive brands" using the US Federal Trade Commission, Massachusetts, and Canadian methods (www.omnicigs.com). These results report reductions in
PAHs from $2 \%$ to $42 \%$, in TSNAs from $1 \%$ to $54 \%$, and in catechols from $15 \%$ to $43 \%$ with Omni. They also reported reductions in nicotine from $17 \%$ to $33 \%$ and changes in carbon monoxide (CO) from $-16 \%$ to $+10 \%$. These results have not been reported in peer reviewed scientific journals.

At the onset of this study, we could not locate published studies of smoking behaviour, substitutability, and toxin exposure in humans using Omni. We tested Omni in smokers over an extended period in their natural environment. Specifically, we were interested in whether the product would: (1) be used similarly to traditional cigarettes; (2) adequately substitute for the pharmacological and behavioural effects of traditional cigarettes; (3) change CO or cotinine levels; and (4) decrease carcinogen exposure.

\section{METHODS}

\section{Funding}

The US National Institutes of Health (NIH) funded this study (see acknowledgements). Neither Vector Group, Ltd (the manufacturer of Omni) nor other tobacco companies provided product or funds.

\section{Design}

We randomly assigned participants to smoke exclusively either their own brand or Omni for six weeks and then crossed them over to the converse condition for the next six weeks (table 1). We chose six weeks because 93\% of the carcinogens we measure are eliminated within six weeks of smoking cessation. ${ }^{9}$ Participants reported cigarettes/day twice a week. Once every two weeks they attended a morning

Abbreviations: CO, carbon monoxide; 1-HOP, 1-hydroxypyrene; IVR, interactive voice recording; PAH, polycyclic aromatic hydrocarbons; PREP, potential reduced exposure product; $\mathrm{NIH}$, National Institutes of Health; NNAL, 4-(methylnitrosamino)-1-(3-pyridyl)-1-butanol and its glucuronides; TSNA, tobacco specific nitrosamines 
laboratory session where they smoked two of the cigarettes they were using during that period with CO levels taken immediately before and after each cigarette. At this visit they also provided a rating of craving and of withdrawal symptoms and provided a 24 hour urine sample for measurement of total nicotine, total cotinine, and two markers of carcinogen uptake: (a) 4-(methylnitrosamino)1-(3-pyridyl)-1-butanol and its glucuronides (total NNAL) which are metabolites of the TSNA 4-(methylnitrosamino)-1(3-pyridyl)-1-butanone (NNK), and (b) 1-hydroxypyrene (1HOP) which is a metabolite of pyrene and a marker of $\mathrm{PAH}$ uptake. The elimination half life of total NNAL is 40-45 days and of 1-HOP is 18 days. ${ }^{9}$ At the end of each six week period participants reported motivation to stop smoking and at the end of the study they completed ratings of Omni.

\section{Participants}

We recruited participants via local newspaper and radio ads that stated: "Smokers wanted for National Institutes of Health-sponsored study of a possibly safer cigarette." Inclusion criteria were: $(1) \geqslant 18$ years old; (2) smoke $\geqslant 10$ cigarettes/day for $\geqslant 1$ year; (3) rate themselves as $<7$ on a 1-10 scale where $1=$ definitely do not intend to quit in the next month and $10=$ definitely intend to quit in the next month; and (4) not pregnant, breastfeeding or planning to become pregnant and have a negative pregnancy test at the onset of the study. All participants read and signed an informed consent that invited smokers "to be in a research study investigating the amount of tar and other chemicals smokers receive with a new cigarette that is said to produce less tar". We reimbursed participants up to $\$ 265$ for their time. The University of Vermont committee on the use of human subjects approved this protocol.

We had difficulty projecting a necessary sample size due to the absence of any data on Omni use and lack of consensus on what would be a clinically significant reduction in carcinogen exposure. We chose a target sample size of 30 participants as likely to be adequate to detect the large effects stated in the Omni website. Initially we planned to recruit only light and ultralight cigarette smokers; however, due to slow recruiting, we also added a group of regular smokers as Omni is available in "full flavour" as well as light varieties (see below).

Among the 115 potential participants screened, we excluded 31. The most common reason for exclusion was planning to quit in next month $(n=12)$. Another 35 were not interested or did not attend the baseline visit, and 15 started but did not complete the study, leaving a sample of 34 smokers. Compared to the sample characteristics of current smokers in the USA as assessed by the 2000 National Health Interview Survey, ${ }^{10}$ our 34 smokers were older ( $48 v 41$ years old), had more men ( $59 \% v 52 \%)$, had fewer minorities ( $3 \% v$ $22 \%)$, had more high school graduates (97\% v 77\%), smoked more cigarettes/day (29v18), and had a earlier age of onset of smoking ( $16 v 18$ years old). Due to our inclusion criteria, the sample consisted of 56\% light/ultralight smokers ( $v 87 \%$ of US smokers) and 53\% precontemplators $/ 47 \%$ contemplators $/ 0 \%$ preparers ( $v 40 \% / 40 \% / 20 \%$ in US smokers). ${ }^{11}$ The sample also had a high mean Fagerstrom test for nicotine dependence score of 6.4 (2.0) versus 4.6 in a recent population based sample of US smokers. ${ }^{12}$ Thirty two participants $(94 \%)$ stated they were interested in purchasing a "safer" cigarette.

\section{Procedures}

Sessions were run in the mornings and we instructed participants to refrain from smoking overnight and for the morning of the session. At the first session, participants completed the Tiffany craving scale $^{13}$ and the Minnesota tobacco withdrawal scale (www.uvm.edu/ hbpl). We provided participants with a two week supply of either their own cigarette or Omni. For light and ultralight smokers we used the Omni Lights (0.8 mg nicotine, $12 \mathrm{mg}$ tar) in either King or $100 \mathrm{~mm}$ length to match the smokers own cigarette (www.omnicigs.com). For regular smokers we used Omni Full Flavor Kings and 100s (1.0 mg nicotine, $15 \mathrm{mg}$ tar). We instructed participants to use the products however they wished, but to use only this tobacco product and to not use any other nicotine or tobacco product or any smoking cessation medication.

We collected a baseline expired air CO level (Vitalograph) and then had the participant smoke one of the assigned cigarettes in their normal manner using a cigarette holder attached to a pneumotachograph (Plowshare Technologies, Baltimore, Maryland, USA). This device used pressure changes to record number of puffs, interpuff interval, and volume/puff. Immediately after completing the first cigarette, a second $\mathrm{CO}$ was obtained. After a 15 minute rest, this procedure was repeated for a second cigarette. We did not use these $\mathrm{CO}$ levels to estimate chronic CO exposure as they were taken in the mornings after overnight abstinence. Rather we used them to measure $\mathrm{CO}$ boost from smoking a cigarette.

At this session, we instructed participants to call twice weekly over the next two weeks to an interactive voice recording (IVR) to enter the number of cigarettes/day. We asked them to save the butts from each cigarette to return at the next session. We also instructed them how to collect a 24 hour urine specimen and asked them to collect urine in the provided specimen bottle for the 24 hours beginning the morning of the day before the next session and ending the morning of the day of the session. At the end of the study, we sent $100 \mathrm{ml}$ samples to the University of Minnesota Cancer Center to be analysed for total cotinine (cotinine plus its glucuronide), total nicotine, total NNAL, and 1-HOP. Total nicotine and total cotinine were analysed as described by

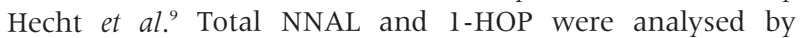
modifications of previously described methods. ${ }^{14-16}$

Table 1 Experimental design*

\begin{tabular}{|c|c|c|c|c|c|c|c|c|c|c|c|c|}
\hline End of week & 1 & 2 & 3 & 4 & 5 & 6 & 7 & 8 & 9 & 10 & 11 & 12 \\
\hline Cigarette & \multicolumn{6}{|c|}{ Omni or own cigarettes } & \multicolumn{6}{|c|}{ Own cigareftes or Omni } \\
\hline Cigarettes/day & $x 2$ & $x 2$ & $x 2$ & $x 2$ & $x 2$ & $\mathrm{X} 2$ & $x 2$ & $x 2$ & $x 2$ & $x 2$ & $x 2$ & $x 2$ \\
\hline Cigarette butts & & $x$ & & $x$ & & $x$ & & $x$ & & $x$ & & $x$ \\
\hline Laboratory smoking & & $x$ & & $x$ & & $x$ & & $x$ & & $x$ & & $x$ \\
\hline Craving, withdrawal & & $x$ & & $x$ & & $x$ & & $x$ & & $x$ & & $x$ \\
\hline 24 hour urine & & $(x)$ & & $(x)$ & & $x$ & & $(x)$ & & $(x)$ & & $x$ \\
\hline SOC & & & & & & $x$ & & & & & & $x$ \\
\hline Omni rating & & & & & & & & & & & & $x$ \\
\hline
\end{tabular}

*Parentheses indicate samples collected but not analysed.

$\mathrm{CO}$, carbon monoxide; NNAL/HOP, carcinogen metabolites; SOC, stage of change. 
At the biweekly sessions, we repeated the above procedures. At the six week visit participants completed a measure of "stage of change" that was modified to not include the 24 hour quit attempt requirement for the preparation stage so that recent changes in motivation to quit could be detected. ${ }^{11}$ Then we provided participants the converse cigarette of what they received in the first six weeks to use for the second six weeks and the procedures were repeated. At the end of the study, participants rated Omni on several attributes.

\section{Data analysis}

We used an analysis of variance (ANOVA) (SAS PROC MIXED) with the following within-participant (paired) factors: type of cigarette (Omni $v$ own), first six week versus second six week period, weeks within each six week period, and first versus second cigarette within the laboratory smoking sessions and the following between-participant factors: light/ultralight versus regular smoker and used Omni versus own cigarette in first six week period. We report any significant $(p<0.05)$ main effects of type of cigarette and interactions between cigarette type and the factors mentioned above. For brevity, we reported only significant $\mathrm{F}$ values.

We based the analysis of cigarettes/day on the 12 IVR ratings taken over each six week period and the number of butts returned at each two week visit. We estimated smoking topography and CO boost based on the two cigs smoked at the three biweekly laboratory visits of each six week period. We next examined substitutability via the craving and withdrawal ratings obtained at the biweekly visits. We then examined nicotine and toxin exposure via analysis of 24 hour urine results based only on the six week and 12 week urine samples. The 2, 4, 8, and 10 week urine sample were not analysed because of financial constraints. We examined motivation to quit based on the modified SOC at the end of each six week period.

\section{RESULTS}

\section{Smoking behaviour}

Self reported cigarettes/day was similar across weeks within the six week periods - that is, no novelty or adaptation effects were observed. Participants smoked a similar number of Omni and own cigs (table 2). Twenty seven of the 34 participants either always returned their cigarette butts or missed but one time. These 27 participants returned a similar number of Omni and own cigarette butts.

In the laboratory smoking sessions, smokers took $9 \%$ fewer puffs (95\% confidence interval (CI) 17\% fewer to 1\% fewer) from the Omni cigarettes $(\mathrm{F}=5.28, \mathrm{p}=0.03)$, but inhaled similar volumes of smoke from each puff of Omni and their

\begin{tabular}{lll} 
Table 2 & Means (SEMs) for study outcomes \\
\hline & Omni & Own cigarette \\
\hline Cigarettes/day & $31(2)$ & $30(2)$ \\
Butts returned & $27(2)$ & $26(2)$ \\
Number of puffs & $11.6(0.5)^{*}$ & $12.7(0.7)$ \\
Volume/puff (ml) & $49(2)$ & $50(2)$ \\
Total puff volume (ml) & $547(25)^{*}$ & $612(34)$ \\
CO boost (ppm) & $+4.8(0.5)^{*}$ & $+4.0(0.5)$ \\
Craving/factor 1 & $4.8(0.2)$ & $4.6(0.2)$ \\
Craving/factor 2 & $2.9(0.2)$ & $2.9(0.2)$ \\
Total withdrawal discomfort & $7.7(0.9)$ & $7.9(0.9)$ \\
Total cotinine (ng/ml/cr) & $4178(400)^{*}$ & $5096(484)$ \\
Total nicotine & $2853(319)$ & $3295(366)$ \\
Total NNAL (pmol/mg/cr) & $2.9(0.3)$ & $3.5(0.4)$ \\
1-HOP (pmol/mg/cr) & $1.5(0.2)$ & $1.7(0.1)$ \\
\hline
\end{tabular}

${ }^{*} \mathrm{p}<0.05$.

$\mathrm{CO}$, carbon monoxide; cr, creatinine; SEM, standard error of the mean; $\mathrm{SOC}$, stage of change. own cigarette. As a result, smokers inhaled $11 \%$ less smoke (that is, total puff volume) (95\% CI $19 \%$ less to $2 \%$ less) from Omni than their own cigarettes $(\mathrm{F}=5.60, \mathrm{p}=0.02)$. The smaller total smoke exposure with Omni was slightly more pronounced in those who smoked their own cigarette first and then Omni $(\mathrm{p}=0.05)$. Despite the smaller smoke exposure, Omni produced a 21\% greater CO boost (95\% CI 3\% greater to $38 \%$ greater) than their own cigarette $(\mathrm{F}=5.74$, $\mathrm{p}=0.02$ ).

When using Omni, smokers reported slightly more craving on the factor 1 scale (intention/desire to smoke and anticipation of pleasure) but this appeared to occur only in those who smoked their own cigarette first and was not significant $(p=0.08)$. Smokers reported no difference in factor 2 craving scores. Smokers reported similar total withdrawal discomfort when using both cigarettes.

Total cotinine was $18 \%$ less ( $95 \%$ CI $31 \%$ less to $5 \%$ less) when using Omni than their own cigarettes $(\mathrm{F}=6.43$, $\mathrm{p}=0.02$.) A similar but non-significant effect was found with urinary total nicotine (13\% less, $95 \%$ CI $31 \%$ less to $4 \%$ more). Total NNAL appeared to be $17 \%$ less (95\% CI $40 \%$ less to $5 \%$ more) with Omni than own cigarettes, but this was not significant. Also, 1-HOP appeared to be $10 \%$ less (95\% CI $28 \%$ less to $7 \%$ more), but again this was not significant.

Six participants had moved forward in their SOC at the end of using Omni and eight at the end of using their own cigarette. At the end of the study, $50 \%$ of participants stated Omni was better for their health than their own cigarette, $71 \%$ stated it was worse tasting, $68 \%$ said it was weaker, and $50 \%$ said it was worse at relieving cravings.

None of the above results differed between light/ultralight smokers who used light Omnis and regular smokers who used full flavour Omnis, nor differed when outliers were or were not excluded.

\section{DISCUSSION}

Our major findings are: (1) Omni may be an adequate substitute for the behavioural and pharmacological effects of traditional cigarettes; (2) even though smokers smoked Omni less intensely and had lower cotinine and nicotine levels, they had had higher CO levels; and (3) Omni did not produce significant declines in two measures of carcinogen exposure.

\section{Smoking behaviour}

After beginning our study, a similar, yet-to-be-published study also tested Omni's ability to substitute for regular cigarettes and, like our study, found no change in cigarettes/ day with Omni. ${ }^{17}$ Our findings that the number of cigarettes/ day was similar between Omni and own cigarettes and that Omni suppressed craving and withdrawal similar to traditional cigarettes suggests Omni can substitute adequately for the behavioural, sensory, and pharmacological effects of traditional cigarettes. On the other hand, in the end-of-study ratings, most smokers stated Omni was worse tasting, weaker, and worse at relieving withdrawal than their own cigarettes. Also, Omni produced cotinine levels that were 18\% less than traditional cigarettes.

Our smokers took fewer puffs with Omni but inhaled each Omni puff similar to that of traditional cigarettes. One possible explanation is that use of the smoking device itself inhibited smoking; however, a recent study suggests using a device similar to ours does not interfere with the external validity of results. ${ }^{18}$ Another possible explanation is that the draw resistance to Omni may be greater than traditional cigarettes. Another possibility is that the taste or sensory characteristics of Omni were stronger or more aversive. The fact that most smokers stated Omni was worse tasting is consistent with this possibility. Another possibility is that Omni produced more concentrated nicotine in smoke; 
however, the fact that Omni use produced lower, not higher, levels of cotinine is inconsistent with this possibility.

Omni produced a greater $\mathrm{CO}$ boost than traditional cigarettes. This was unexpected given that the total smoke exposure/cig was smaller with Omni. In machine testing, CO levels with Omni ranged from $16 \%$ less to $10 \%$ more than comparison cigarettes (www.omnicigs.com). In addition, the Omni study that began after our study did not measure CO boost; however, it did report lower, not higher, CO when levels were taken during the day. ${ }^{17}$

One possible explanation for the increased CO in our study is that the filter on Omni does not dilute $\mathrm{CO}$ as much as traditional cigarette filters. Another possibility is that the burning characteristics of the modified tobacco promote more incomplete combustion. A third possibility is that vent blocking increased $\mathrm{CO}$ even though smoking topography was less. Since CO has been linked to cardiovascular disease, ${ }^{19} 20$ and since cardiovascular disease accounts for as many deaths from smoking as cancer, ${ }^{21}$ further studies are needed to determine if Omni reliably increases CO levels.

\section{Toxin exposure}

After six weeks in humans, we found reductions of $17 \%$ for total NNAL and $10 \%$ for 1 -HOP; however, neither reduction was statistically reliable. In machine tests, Omni reduced NNK ( the parent compound for NNAL) by 53-66\% depending on testing method (www.omnicigs.com) and pyrene by 20 $29 \%$. The study of Omni that began after ours reported a $21 \%$ reduction in NNAL that was significant and 5\% reduction in 1-HOP that was not significant. ${ }^{17}$

One possible reason for our failure to find significant differences is our small sample size. Given the within-subject variability we observed, our study had $80 \%$ power to detect a significant decline in total NNAL if the true decline was $1.1 \mathrm{pmol} / \mathrm{mg} / \mathrm{cr}(-32 \%)$ and in $\mathrm{l}-\mathrm{HOP}$ if the true decline was $0.42 \mathrm{pmol} / \mathrm{mg} / \mathrm{cr}(-25 \%)$ with Omni. Unfortunately, given the expense of carcinogen analysis, our funds did not allow a larger sample size.

The lower than expected reduction in urinary total NNAL in our study may be due to non-compliance. Few of the cigarette butt returns were incomplete; however, we did not otherwise objectively verify use of cigarettes. The lower amount of reduction may also be due to differences in smoking topography in our subjects versus the machine smoking conditions under which NNK were measured. Levels of total NNAL (the metabolite of NNK) in the urine of our smokers would not have been affected by non-tobacco exposures as NNK is quite tobacco specific; however, levels of 1 -HOP are affected by exposure to pyrene through the diet.' Since diet was not controlled in this study, this could have confounded the 1-HOP results. Also, if we had selected only smokers with currently high levels of NNAL and 1-HOP, we may have found reliable and perhaps larger differences.

Our carcinogen results can be interpreted in several ways. Promoters of harm reduction would point out that given the high prevalence of smoking, ${ }^{22}$ the large toxicity of smoking, ${ }^{21}$ and that less than half of smokers quit smoking, ${ }^{22}$ even a small decrease in carcinogen exposure could have a large public health impact. Sceptics would doubt whether 10-17\% reductions in carcinogen metabolite levels are sufficiently large to have a health impact. In addition, they would point out that many other carcinogens, some of which may be more important than NNK and PAH, were not examined. Finally, they would be concerned that claims of less exposure or risk based on these results would undermine motivation for cessation and may increase initiation of smoking. Our own interpretation is that our results neither support nor totally refute Omni claims of reduced exposure or risk for cancer.

\section{What this paper adds}

The tobacco industry has marketed several cigarettes that either explicitly or implicitly claim to reduce the harm from smoking. We tested a cigarette that has advertised large reductions in certain carcinogens-Omni. During a six week period, smokers smoked this new cigarette less intensely than their own cigarette yet had higher carbon monoxide levels from smoking. Carcinogen levels appeared to decrease slightly but not significantly. With our small sample size, it is unclear if there is absolutely no decrease in carcinogens; however, we could not confirm the large decrease in carcinogens reported by the industry. Our negative findings illustrate the pressing need to have a governmental body regulate claims of "less risky" cigarettes.

In summary, our results suggest Omni appears to be a product that would be acceptable to smokers; however, its production of increased $\mathrm{CO}$ and its failure to produce reliable and large reductions in carcinogens is disappointing. Given the above, we believe its claims of less exposure or risk are unwarranted until adequate independent studies can confirm decreased exposure or risk in humans and until studies show no harm from such claims on smoking cessation and initiation. This is especially important given the widespread advertising for PREPs and the lack of a willingness by governments to regulate these devices.

\section{ACKNOWLEDGEMENTS}

This study was funded by NIDA grants DA-11935 (JH), and DA13333 (SH) and NIDA Senior Scientist Award DA-00490 (JH). We thank Jonathan Hirsch, Josue Keely and Elizabeth Iula for help in conducting the study and Irene Kramarczuk and Mikel Roe for technical assistance with the biological analyses.

\section{Authors' affiliations}

J R Hughes, University of Vermont, Departments of Psychiatry, Psychology and Family Practice, Burlington, Vermont, USA

S S Hecht, S G Carmella, S E Murphy, University of Minnesota Cancer Center, Minneapolis, Minnesota, USA

P Callas, University of Vermont, Biometry Facility, Burlington, Vermont, USA

\section{REFERENCES}

1 Shiffman S, Gitchell JG, Warner KE, et al. Tobacco harm reduction: conceptual structure and nomenclature for analysis and research. Nicotine Tob Res 2002;4:S113-29.

2 Institute of Medicine. Clearing the smoke. Assessing the science base for tobacco harm reduction. Washington DC: National Academy Press, 2001.

3 Warner KE. Tobacco harm reduction: promise and perils. Nicotine Tob Res 2002:4:S61-71.

4 Hatsukami DK, Slade J, Benowitz NL, et al. Reducing tobacco harm: research challenges and issues. Nicotine Tob Res 2002;4:S89-101.

5 World Health Organization. Monograph. Advancing knowledge on regulating tobacco products. Helsinki, Finland: World Health Organization, 2001.

6 Fairclough G. Vector produces nicotine-free cigarette using genetically modified tobacco. The Wall Street Journal January 16, 2001.

7 Fairclough G. Smoking rivalry. The race to produce a 'safer' cigarette shifts to high gear. The Wall Street Journal February 13, 2001.

8 Hecht SS. Human urinary carcinogen metabolites: biomarkers for investigating tobacco and cancer. Carcinogenesis 2002;23:907-22.

9 Hecht SS, Carmella SG, Chen M, et al. Quantitation of urinary metabolites of a tobacco-specific lung carcinogen after smoking cessation. Cancer Res 1999;59:590-6.

10 Hughes JR. Data to determine generalizability of tobacco research. Nicotine Tob Res, (in press).

11 Spencer L, Pagell F, Hallion ME, et al. Applying the transtheoretical model to tobacco cessation and prevention: a review of literature. Am J Health Promotion 2002;17:7-71.

12 Shiffman S, Pillitteri JL, Burton SL, et al. Smokers' beliefs about 'light' and 'ultra light' cigarettes. Tobacco Control 2001;10:i23-7.

13 Tiffany ST, Drobes DJ. The development and initial validation of a questionnaire on smoking urges. Br J Addiction 1991;86:1467-76. 
14 Carmella SG, Han S, Fristad A, et al. Improved method for analysis of total 4 (methylnitrosamino)-1-(3-pyridyl)-1-butanol (NNAL) in human urine. Cancer, Epidemiology and Biomarkers Preventive (in press).

15 Carmella SG, Akerkar SA, Richie JP Jr, et al. Intraindividual and interindividual differences in metabolites of the tobacco-specific lung carcinogen 4-(methylnitrosamino)-1-(3-pyridyl)-1-butanone (NNK) in smokers' urine. Cancer, Epidemiology and Biomarkers Preventive 1995:4:635-42.

16 Jongeneelen FJ, Anzion RBM, Henderson PT. Determination of hydroxylated metabolites of polycyclic aromatic hydrocarbons in urine. J Chromatogr 1987;413:227-32.

17 Lemmonds C, Murphy S, Hecht S, et al. Modified tobacco products. Effects on risk factors for disease. Paper presented at the Annual Meeting of the Society for Research on Nicotine and Tobacco. New Orleans, February, 2003.
18 Lee EM, Malson JL, Waters AJ, et al. Smoking topography: Reliability and validity in dependent smokers. Nicotine Tob Res 2003;5:673-9.

19 Heliovaara M, Karvonen MJ, Vilhunen R, et al. Smoking, carbon monoxide, and atherosclerotic diseases. BMJ 1978;i:268-70.

20 Wald N, Howard S, Smith PG, et al. Association between atherosclerotic diseases and carboxyhaemoglobin levels in tobacco smokers. BMJ 1973;i:761-5.

21 US Department of Health and Human Services. The health benefits of smoking cessation. A report of the Surgeon General, 1990. Rockville, Maryland: Public Health Service, Centers for Disease Control, Office on Smoking and Health, 1990. (DHHS Publication No (CDC) 90-8416.)

22 Jha P, Ranson MK, Nguyen SN, et al. Estimates of global and regional smoking prevalence in 1995, by age and sex. Am J Public Health 2002;92:1002-6. 